\title{
Tribological Performance of Textured 316L Stainless Steel Prepared By Selective Laser Melting*
}

Ping Chen ( $\square$ chenp@ustb.edu.cn )

University of Science and Technology Beijing https://orcid.org/0000-0003-4925-3976

\section{Xiaojie Liu}

University of Science and Technology Beijing

Kangning Jin

University of Science and Technology Beijing

Xiaoxi Qiao

University of Science and Technology Beijing

Weixu Yang

University of Science and Technology Beijing

\section{Original Article}

Keywords: SLM, Surface texture, Tribological performance, 316L SS

Posted Date: August 31st, 2021

DOI: https://doi.org/10.21203/rs.3.rs-829565/v1

License: (c) (i) This work is licensed under a Creative Commons Attribution 4.0 International License. Read Full License 


\title{
Tribological performance of textured 316L stainless steel prepared by
}

\author{
selective laser melting* \\ Ping Chen ${ }^{1,2 \dagger}$, Xiaojie Liu ${ }^{1}$, Kangning Jin ${ }^{1}$, Xiaoxi Qiao ${ }^{1,2}$, Weixu Yang ${ }^{1,2}$ \\ ${ }^{1}$ School of Mechanical Engineering, University of Science and Technology Beijing, \\ Beijing 100083, China; \\ ${ }^{2}$ Shunde Graduate School of University of Science and Technology Beijing, Shunde \\ 528300, China
}

\begin{abstract}
Selective laser melting (SLM) technology is a rapid prototyping additive manufacturing technology, which is widely used in the biomedical field. Surface texturing technology, as a surface treatment technology, has been used to improve the tribological performance. In the paper, both SLM and surface texturing technology are used to prepare textured samples with different parameters. Under the conditions of dry and phosphate buffer solution (PBS) lubricated conditions, the tribological performance and the mechanism of the textured 316L SS were investigated. The texture parameters studied are characteristic size, area ratio and shape including single triangular, circular and composite textures composed of two shapes. Results show that under dry friction condition, compared with the untextured surfaces, the friction coefficients and wear losses of the three textured samples almost increased, and only the wear loss of the circular textured sample decreases. Under the condition of PBS solution, compared with the untextured surface, most of the friction coefficients and wear loss of the textured samples decreased significantly, but the wear loss of textured surfaces in group B shows an obvious increase. And reducing the characteristic size of the texture significantly increases the wear loss of the $316 \mathrm{~L} \mathrm{SS}$ sample. At the same time, the triangular textured samples have better wear resistance than the circular textured samples. Therefore, the application of surface texturing technology to 316L SS samples prepared by SLM, especially in the simulated body fluid conditions, can achieve the purpose of stable friction and reduce wear loss. Meanwhile, reasonable texture parameters have a greater impact on improving the tribological performance of 316L SS.
\end{abstract}

Keywords: SLM; Surface texture; Tribological performance; 316L SS

PACS: $81.40 . \mathrm{Pq}, 46.55 .+\mathrm{d}$

\section{Introduction}

Selective Laser Melting technology (SLM) is a rapid prototyping additive manufacturing technology by using the powdered metal or plastic to print parts layer by layer. SLM has the advantages of personalized printing and rapid prototyping for the

\footnotetext{
* Project supported by the National Natural Science Foundation of China (Grant No. 51975042 and 51905032), the Science and Technology Plan Project of Guangdong Province (Grant No. 2019B010150001), and the Fundamental Research Funds for the Central Universities, China (Grant No. FRF-TP-19-004A3).

${ }^{+}$Corresponding author. E-mail: chenp@ustb.edu.cn
} 
production of complex parts with unique processing method, and it is widely used in aerospace, food processing, biomedical equipment, and automotive electronics [1-3]. At present, the method of using SLM to fabricate biomedical implant components has attracted extensive attention in various research fields. Studies show that SLM technology has more outstanding advantages in preparing parts comparing with the traditional processing methods [4-6], such as higher wear resistance. The biomedical implant prepared by SLM has a unique porous structure, and the rough surface can solve the stress shielding phenomenon of the medical implant during using. Moreover, it is beneficial to promote the generation of new bone tissue in the implant, and plays a role in fixing the implant and the body's bone.

316L stainless steel (316L SS) is a widely used biomedical material, which can be used for the preparation of fracture plates and hip nails for internal repair of various medical devices and implants, especially as the medical implants [7]. Studies have shown that the performance of 316L SS prepared by SLM differs greatly from that of 316L SS prepared by traditional technology [8-11]. Under the condition of oil lubrication, Y. Zhu et al. [9] found that the friction coefficient of 316L SS prepared by SLM was smaller than that of $316 \mathrm{~L}$ SS fabricate by traditional technology, and the surface topography is smoother. Under the dry friction condition, Y. Sun et al. [10] found that the wear rate of $316 \mathrm{~L}$ SS prepared by SLM is greater than that of hot-rolled 316L SS through pin-disk experiments, which is related to the porosity of 316L SS. Yi Zhu et al. [11] studied the effect of porosity on tribological performance of 316L SS prepared by SLM. Under oil lubrication conditions, the friction coefficients of 316L SS decreased with the increase of porosity. This is main reason is that the pores can store oil and provide additional hydrodynamic lubrication effects, which improves the tribological performance of the 316L SS. However, 316L SS prepared by SLM has unavoidable defects such as wear and fracture during its use [12]. Therefore, the tribological performance of $316 \mathrm{~L} \mathrm{SS}$ need to be further optimized.

Surface texturing technology is a surface treatment technology in which small grooves or protrusions with specific size, arrangement and shape are processed on the solid surface. It is mostly used for the modification and optimization of the material 
surface. Many studies have shown that surface texturing technology can play an important role in reducing wear and friction, and improving surface lubrication performance. Above all, the texture parameters have different effects on the tribological performance of metal materials. The tribological performance were studied by applying the surface texturing technology to the stamping die $[13,14]$, the results showed that the wear weight of the textured die was relatively slight compared to the untextured die, and the friction coefficient was smaller. It was found that [15], when the friction direction slid from the equilateral to the diagonal direction of the triangular texture, the triangular textured samples had obvious hydrodynamic lubrication effect comparing to the circular and square textured samples, for the three textured samples with the same area ratio. Meanwhile, the composite textured surface has the better tribological performance than the textured surface with a single shape [16]. Dawit Zenebe Segu [17] experimentally studied the friction coefficient and wear weight of composite textured samples (circular and oval) with different area ratios, and found that the friction coefficients of composite textured surfaces were lower and more stable than that of untextured surface.

In this study, both the combination of surface texturing technology and SLM technology were used to print textured biomedical samples at one time during the SLM process. Not only can reduce the complexity of different process conversions in the preparing process, but also combine the outstanding advantages of surface texturing technology in the field of tribology. The tribological performance of 316L SS under dry friction and simulated body fluid (PBS) conditions were explored by using the biomedical materials 316L SS in vitro dry environment and vivo liquid environment. It provides experimental basis and theoretical guidance for the application of textured 316L SS prepared by SLM in the field of biomedicine.

\section{Experimental details}

\subsection{Materials and texture parameters}

According to the relevant standards of SLM technology for metal powders [18], the particle size of the powder ranges from $20 \mu \mathrm{m}$ to $50 \mu \mathrm{m}$. Meanwhile, the powder is required to have the advantages of high sphericity, high fluidity and low oxygen content. 
The 316L SS powder used in this study contains Fe, Cr, Ni, Mo, Si, Mn and other elements. Its element content is listed in Table 1, and its particle size distribution and micromorphology are shown in Fig. 1. It can be seen that the particle size of the powder meets standards, and the particle size of $35.5 \mu \mathrm{m}$ accounts for about $25 \%$ of the mass fraction, reaching the maximum. It can be seen from the SEM images of the powder in Fig. 1(b) that most of the powder is approximately spherical.

Table 1 Elemental content of 316L SS powder.

\begin{tabular}{cccccccccc}
\hline Elements & $\mathrm{Fe}$ & $\mathrm{C}$ & $\mathrm{Si}$ & $\mathrm{Mn}$ & $\mathrm{S}$ & $\mathrm{P}$ & $\mathrm{Cr}$ & $\mathrm{Ni}$ & $\mathrm{Mo}$ \\
\hline \multirow{2}{*}{ Content } & Bal. & $\leqslant$ & $\leqslant$ & $\leqslant$ & $\leqslant$ & $\leqslant$ & & & $\leqslant$ \\
& & 0.03 & 1.0 & 2.0 & 0.03 & 0.035 & $16.0 \sim 18.0$ & $10.0 \sim 14.0$ & $2.0 \sim 3.0$ \\
\hline
\end{tabular}
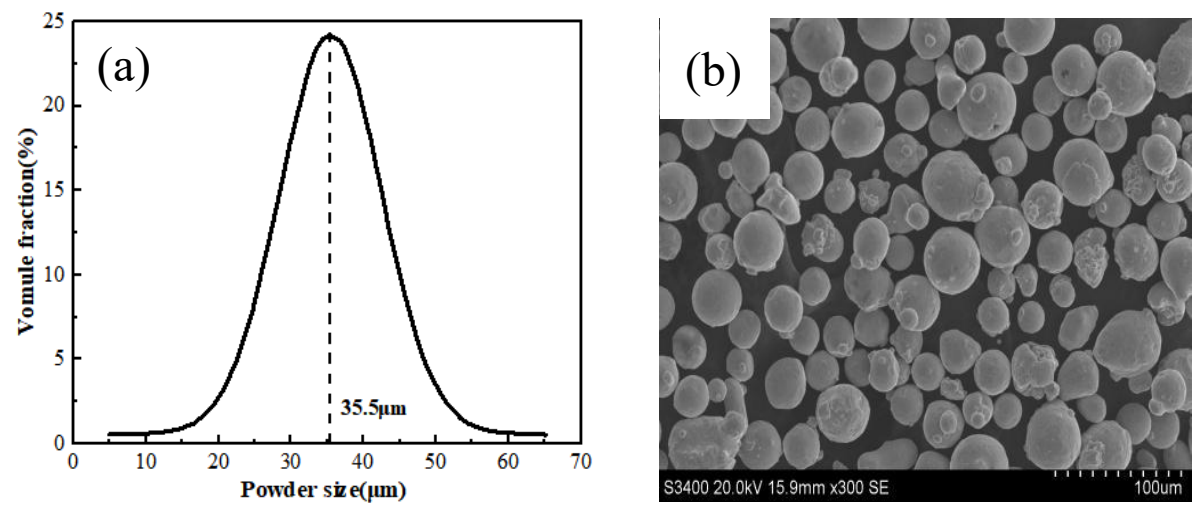

Fig.1. 316L SS powder particles: (a) size distribution; (b) micromorphology.

The characteristic size (the side length of a regular triangle or the diameter of a circle), area ratio and shape of the texture were studied, where the textured depth was $100 \mu \mathrm{m}$. The parameters of the textured surfaces prepared by the SLM process are listed in Table 2, which are divided into 3 groups. Among them, the B group reduces the characteristic size of the texture relative to the A group, the $\mathrm{C}$ group decreases the area ratio of the texture relative to the A group, and the $\mathrm{C}$ group decreases to a greater extent. For comparison, untextured samples by SLM process. 
Table 2 Texture parameters.

\begin{tabular}{|c|c|c|c|c|}
\hline Group & Distribution & Characteristic size & Area ratio & Samples / Shape \\
\hline A & & $\begin{array}{l}\text { Regular triangle with } \\
\text { length of } 600 \mu \mathrm{m} \text {; } \\
\text { Equal-area circle with } \\
\text { diameter of } 445 \mu \mathrm{m} \text {. }\end{array}$ & $20 \%$ & $\begin{array}{l}\text { A1 / Triangle } \\
\text { A2 / Circle } \\
\text { A3 / Triangle and circle }\end{array}$ \\
\hline B & & $\begin{array}{l}\text { Regular triangle with } \\
\text { length of } 550 \mu \mathrm{m} \text {; } \\
\text { Equal-area circle with } \\
\text { diameter of } 408 \mu \mathrm{m} \text {. }\end{array}$ & $20 \%$ & $\begin{array}{l}\text { B1 / Triangle } \\
\text { B2 / Circle } \\
\text { B3 / Triangle and circle }\end{array}$ \\
\hline $\mathrm{C}$ & 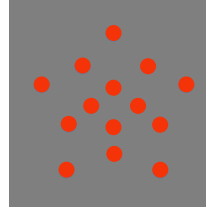 & $\begin{array}{l}\text { Regular triangle with } \\
\text { length of } 600 \mu \mathrm{m} \text {; } \\
\text { Equal-area circle with } \\
\text { diameter of } 445 \mu \mathrm{m} \text {. }\end{array}$ & $10 \%$ & $\begin{array}{l}\mathrm{C} 1 / \text { Triangle } \\
\mathrm{C} 2 \text { / Circle } \\
\text { C3 / Triangle and circle }\end{array}$ \\
\hline
\end{tabular}

\subsection{Samples preparation}

An EP-M100T metal 3D printer was used to prepare the 316L SS samples, and the schematic diagram of SLM manufacturing metal parts is shown in Fig. 2. Before the laser starts to scan, the horizontal powder spreader rolls the metal powders onto the substrate of the forming chamber, and then the laser beam selectively melts the powders on the substrate according to the contour information to form the current layer. The lifting system lowers the height of a powder layer, and the powder spreader rolls the metal powder on the already formed layer. Then the laser beam melts the new metal powders, and the equipment is transferred to the next layer. The process is repeated until the entire part is prepared. The whole process is carried out in a forming chamber with a protective gas-argon to avoid reaction with other gases under high temperature conditions. 


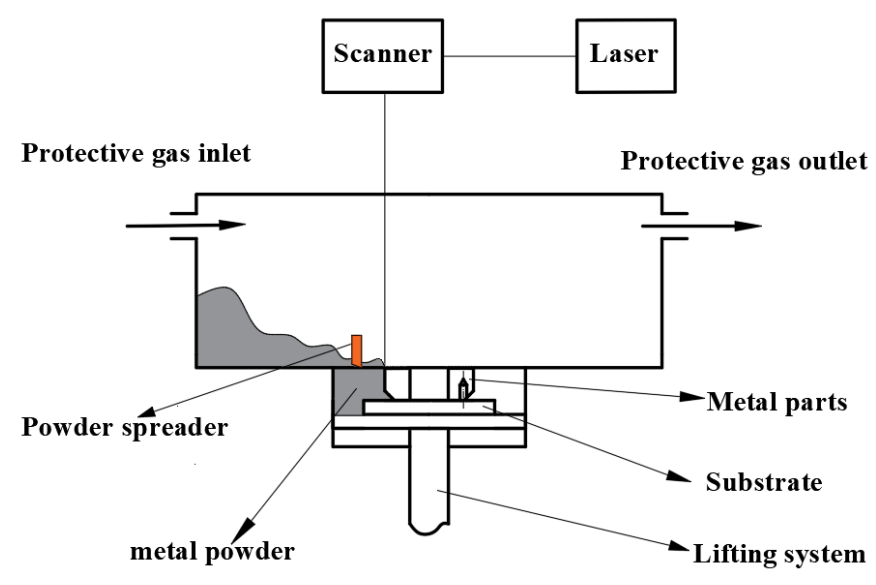

Fig. 2. Schematic of SLM forming principle.

In the SLM process of preparing samples, the most important parameters are laser scanning power, hatch spacing and scanning speed. According to Ref.[19], in the SLM process of preparing 316L SS samples, when the input power density value is between $50 \mathrm{~J} / \mathrm{mm}^{3}$ and $70 \mathrm{~J} / \mathrm{mm}^{3}$, the quality of the sample is the best. Therefore, before studying tribological performance, the SLM process parameters were studied by changing the scanning speed to improve the quality of the 316L SS samples. The SLM process parameters used are listed in Table 3 , and the power density used was $70 \mathrm{~J} / \mathrm{mm}^{3}$ obtained according to the equation (1).

Table 3 SLM process parameters

\begin{tabular}{cccccc}
\hline Parameters & Hatch spacing & Power & Scanning speed & Layer thickness & Scan type \\
\hline Value & $80 \mu \mathrm{m}$ & $80 \mathrm{~W}$ & $700 \mathrm{~mm} / \mathrm{s}$ & $20 \mu \mathrm{m}$ & Checkerboard \\
\hline \multirow{5}{*}{$w=\frac{p}{v \times h \times t}=\frac{80}{700 \times 0.08 \times 0.02}$} & $70\left(\mathrm{~J} / \mathrm{mm}^{3}\right)$ &
\end{tabular}

Where $p$ is the power, $v$ is the scanning speed, $h$ is the hatch spacing, $t$ is the layer thickness and $w$ is the power density.

The $316 \mathrm{~L}$ SS samples were prepared into the $20 \times 20 \times 6 \mathrm{~mm}^{3}$ cuboid. The texture was distributed in the annular region on the upper surface of the bottom sample with the inner diameter of $7 \mathrm{~mm}$ and the outer diameter of $15 \mathrm{~mm}$, as Fig. 3(a) shows. The detail parameters of the surface textures are given in the Table 2 . 


\subsection{Friction and wear tests}

The CFT-I material surface property comprehensive tester was used to study the tribological performance of the textured $316 \mathrm{~L} \mathrm{SS}$. And the schematic diagrams of the lower sample and the friction pair used in the tester are shown in Fig. 3. The upper sample is the CGr15 ball, which is fixed on the tester, and has a diameter of $4 \mathrm{~mm}$. The lower sample is the 316L SS sample, which is fixed by a ring with an inner diameter of $15 \mathrm{~mm}$. During the experiment, the rotation radius was set to $5 \mathrm{~mm}$. Test parameters are: rotation speed $200 \mathrm{r} / \mathrm{min}$, load $20 \mathrm{~N}$, test time $30 \mathrm{~min}$. The average values and standard deviations of three repeatability tests were calculated for analysis. All tests were carried out in ambient temperature $\left(22^{\circ} \mathrm{C}\right)$ and relative humidity $(18 \% \mathrm{RH})$.

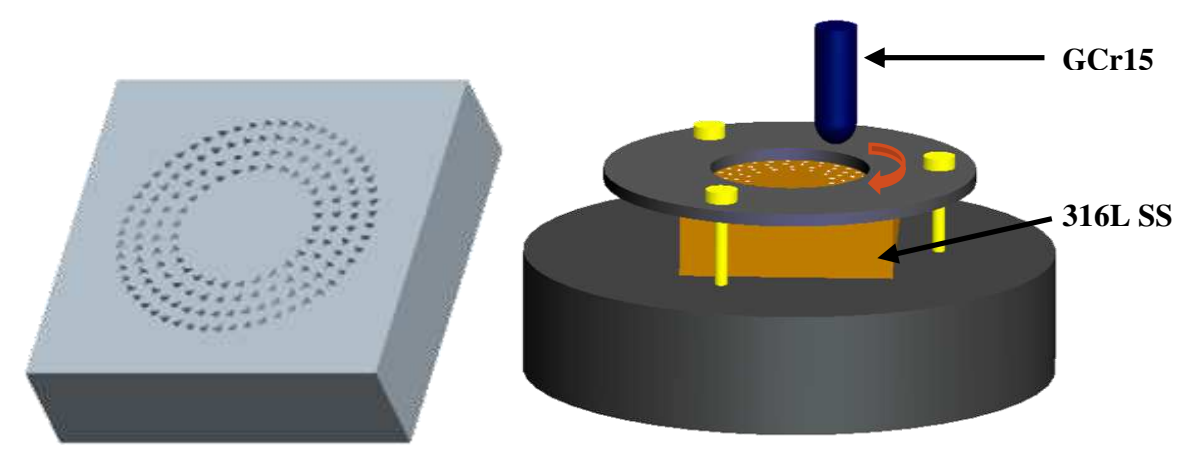

Fig. 3. Schematic diagrams of (a) the tested samples and (b) friction pair

Before the tests, the samples were performed a sandblasting treatment to remove oxide film, sticky sand and rust on the sample surfaces. In order to ensure the same surface finishes, the contacting surfaces of all samples were sanded with 2000\# sandpaper. The samples were cleaned with acetone solution in the ultrasonicator for $10 \mathrm{~min}$ and rinsed with deionized water for $30 \mathrm{~s}$ before and after the tests. The samples are immersed in the phosphate buffer solution (PBS) for $1 \mathrm{~h}$ to simulate the body fluid conditions ( $\mathrm{pH}=7.2$ ), and the chemical composition of PBS solution is listed in Table 4. The average friction coefficient in the final stable friction stage is calculated, and the wear loss is obtained by measuring the weight difference of the sample before and after the experiments. The Hitachi s-3400N electron microscope was used for scanning electron microscopy (SEM) of the sample surface morphology. 
Table 4 Chemical composition of PBS.

\begin{tabular}{ccccc}
\hline Ingredient & $\mathrm{NaCl}$ & $\mathrm{KCl}$ & $\mathrm{KH}_{2} \mathrm{PO}_{2}$ & $\mathrm{NaH}_{2} \mathrm{PO}_{2}$ \\
\hline Concentration percentage $(\mathrm{g} / \mathrm{L})$ & 8.0 & 0.2 & 0.24 & 1.42 \\
\hline
\end{tabular}

\section{Results and discussion}

\subsection{Characterization of textured surfaces}

The untextured and textured 316L SS samples prepared by SLM technology are shown in Fig. 4. And the SEM images of the textured sample with the textured parameter of B group are shown in Fig. 5. There are defects on the surface of the triangular textured sample in Fig. 5(a), which cannot be completely eliminated after grinding. Moreover, the triangular texture tends to be irregular, especially the smooth transitions are prone to occur at sharp corners of the triangle. By comparing and analyzing, it can be seen that the surface of the circular textured sample shown in Fig. 5(b) is relatively flat, with fewer defects and the best surface morphology. The surface of the composite textured sample is rougher than the surface of the single textured sample (Fig. 5(c)). This is due to the shape of the composite textured sample is irregular during the SLM processing, which causes more defects to affect the textured morphology. Meanwhile, there are many defects near the composite texture, such as unmelt powder particles, and the bonding strength with the matrix is not high. These unstable solid particles are very easy to fall off during the friction process, which increases the abrasive wear between materials, resulting in furrows on the sample surface [20]. The comparison of the actual and theoretical characteristic size values of the different texture parameters are listed in Table 5. Among them, the reduction percentage in characteristic size of the circular textured sample is smaller than that of the triangular textured sample. Compared with the corresponding shape of the single textured sample, the reduction percentage of the composite textured sample increases. This means that under the same friction conditions, the circular textured sample has a larger volume to store the wear debris than the triangular textured sample. 


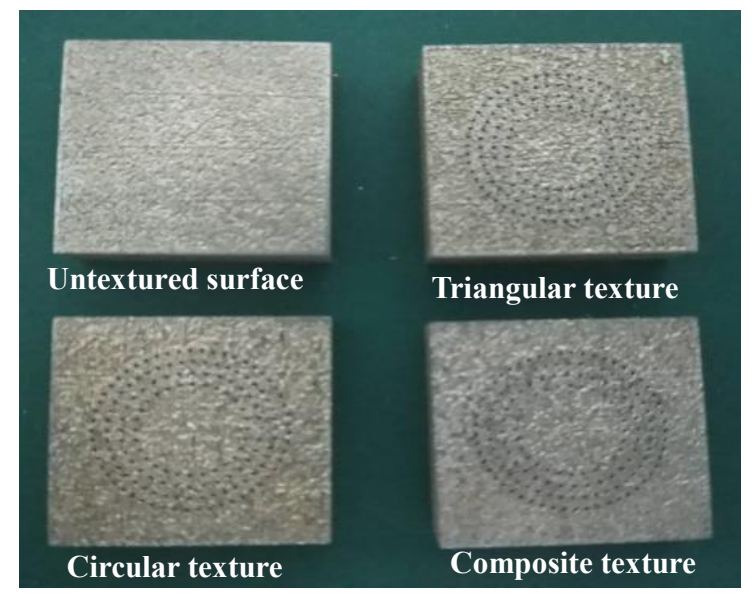

Fig. 4. 316L SS samples prepared by SLM
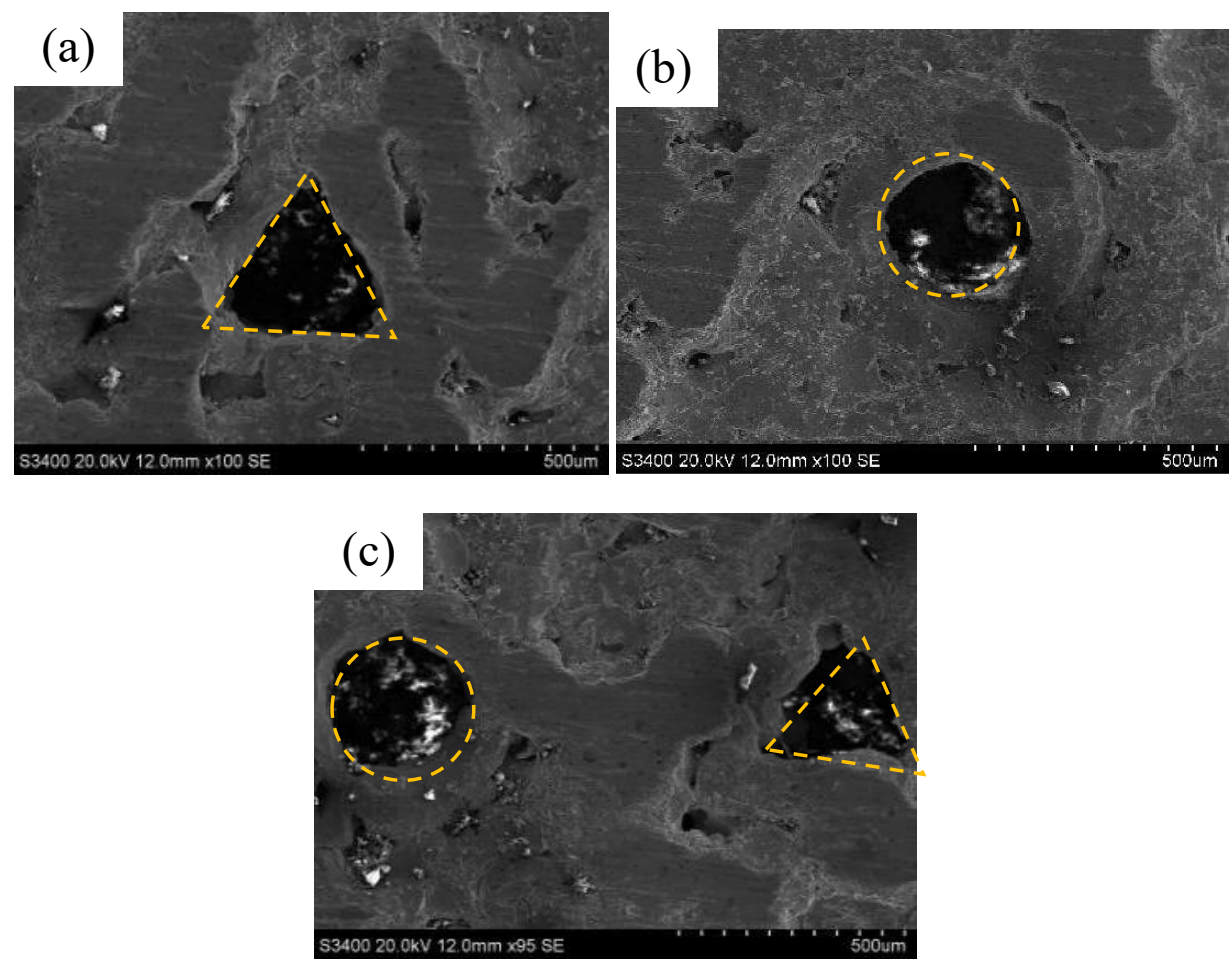

Fig. 5. SEM images of (a) triangular, (b) circular, and (c) composite textured samples.

Table 5 Actual characteristic size of texture.

\begin{tabular}{ccccc}
\hline \multirow{2}{*}{ Textured shape } & $\begin{array}{c}\text { Theoretical } \\
\text { characteristic size }\end{array}$ & $\begin{array}{c}\text { Actual } \\
\text { characteristic size }\end{array}$ & $\begin{array}{c}\text { Reduction } \\
\text { percentage (\%) }\end{array}$ \\
\hline \multirow{2}{*}{ Single texture } & Triangle & 600 & 380 & 36.67 \\
& Circle & 445 & 300 & 32.58 \\
\multirow{2}{*}{ Composite texture } & Triangle & 600 & 360 & 40.00 \\
& Circle & 445 & 285 & 35.96 \\
\hline
\end{tabular}




\subsection{Tribological performance under dry friction conditions}

The friction coefficients of textured samples with different texture parameters under dry friction conditions are shown in Fig. 6, and the yellow dotted line indicates the friction coefficient of the untextured sample prepared by SLM. As a result, the contact between the GCr15 ball and the 316L SS sample was mainly a point-point high pair, and the contact point was subject to large forces, so the friction coefficient was large and unstable. As the friction process continuing, the top of the rough peak was gradually worn away, and the surface roughness decreased. Since the actual contact area of the friction pair increased, the friction coefficient decreased and changed steadily, and friction condition gradually entered into a stable stage. The friction coefficients of the circular textured sample in group A and the untextured sample were basically the same at the stable friction stage, and the friction coefficient of the circular textured sample was finally stable at about 0.44 . The friction coefficient of the composite textured sample and that of the triangular textured sample remained basically the same and were relatively larger than that of the circular textured sample, but the friction coefficient of the composite textured sample fluctuated relatively. The friction coefficients of textured samples in group B (with the reducing textured characteristic size) increased significantly and the fluctuation increases during the friction process. At the same time, the friction coefficient of the circular textured samples was increased by about 0.18 compared with the untextured sample. The friction coefficient of the triangular textured sample was almost same with that of the circular textured sample. Similarly, the friction coefficient of the composite textured sample was the largest, which was about 0.9 at the stable friction stage. The friction coefficients of the textured samples in group $\mathrm{C}$ obtained by reducing the textured area ratio showed a slight upward trend. The friction coefficient of the circular textured sample increased by about 0.04 compared to that of the untextured sample, which fluctuated relatively little. In the final stable friction stage, the friction coefficient of the triangular textured sample was stable at 0.52 , and the friction coefficient of the composite textured sample remained the largest and fluctuated significantly. 


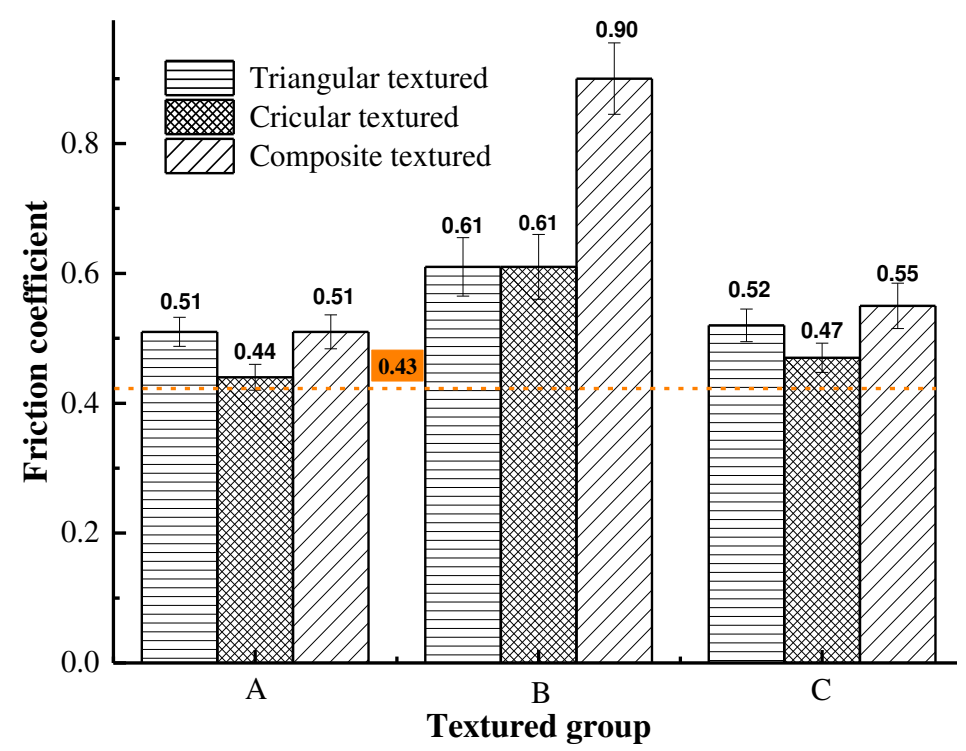

Fig. 6. The friction coefficients of textured samples with different texture parameters under dry friction conditions.

The wear loss of the textured samples is shown in Fig. 7. The yellow dotted line indicates the wear loss of the untextured sample prepared by SLM. On the whole, the wear loss kept the same change law with the friction coefficient: composite textured samples $>$ triangular textured samples $>$ untextured samples $>$ circular textured samples. During the friction process, the reduced ability to store wear debris for the composite textured sample with small actual textured characteristic size will accelerates the wear loss of the material, and thus leads to the most severe wear of the composite textured sample. For the single textured sample, the texture mainly played the role of storing wear debris, so the wear resistance was improved. Moreover, the circular texture has a larger volume than the triangular texture and can store more wear debris. Reducing the characteristic size or the area ratio of the texture would cause the wear loss to increase by comparing the wear loss of 3 groups of samples. This is mainly due to reducing the ability of the texture to store wear debris under dry friction conditions, which causes more wear debris generated in the friction process to participate in the new friction and wear process, increasing the probability of three-body friction and reducing wear resistance of the sample surface. 


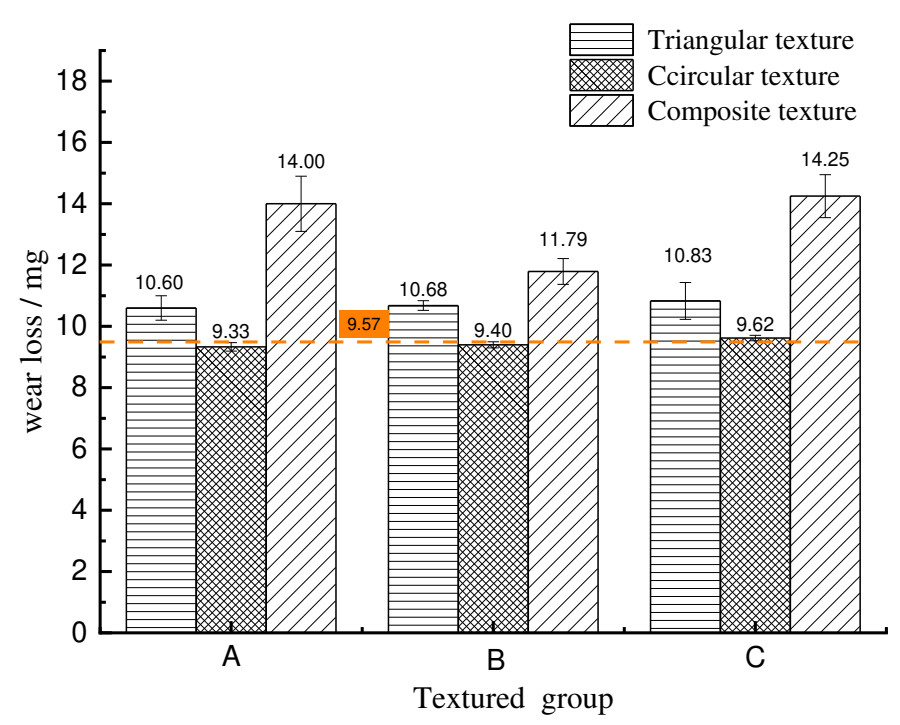

Fig. 7. The wear loss of textured samples with different texture parameters under dry friction conditions.

Further, the surface morphology of the tested samples prepared by SLM were analyzed, as shown in Fig. 8., It can be seen that obvious crack failure occurred on the untextured sample surface (Fig 8(a)).And there were spherical small particles inside the crack, as shown in the enlarged image of Fig. 8(b), indicating that the crack was formed during SLM processing. During the friction, Crack became brittle, and it accelerated the crack extension and led to a more severe flaking of the sample surface. For the circular textured samples, there were a lot of wear debris inside the texture, as shown in Fig. 8(c) and 8 (d). The texture on the surface had been basically filled, and only the general outline of the texture could be seen in Fig. 8(c). The existence of lamellar wear debris inside the texture indicated that the existence of the texture did play a role in storing the wear debris and reducing the wear caused by the wear debris (Fig. 8(d)). This can explain the effect of texture parameters on the wear loss to a certain extent. 

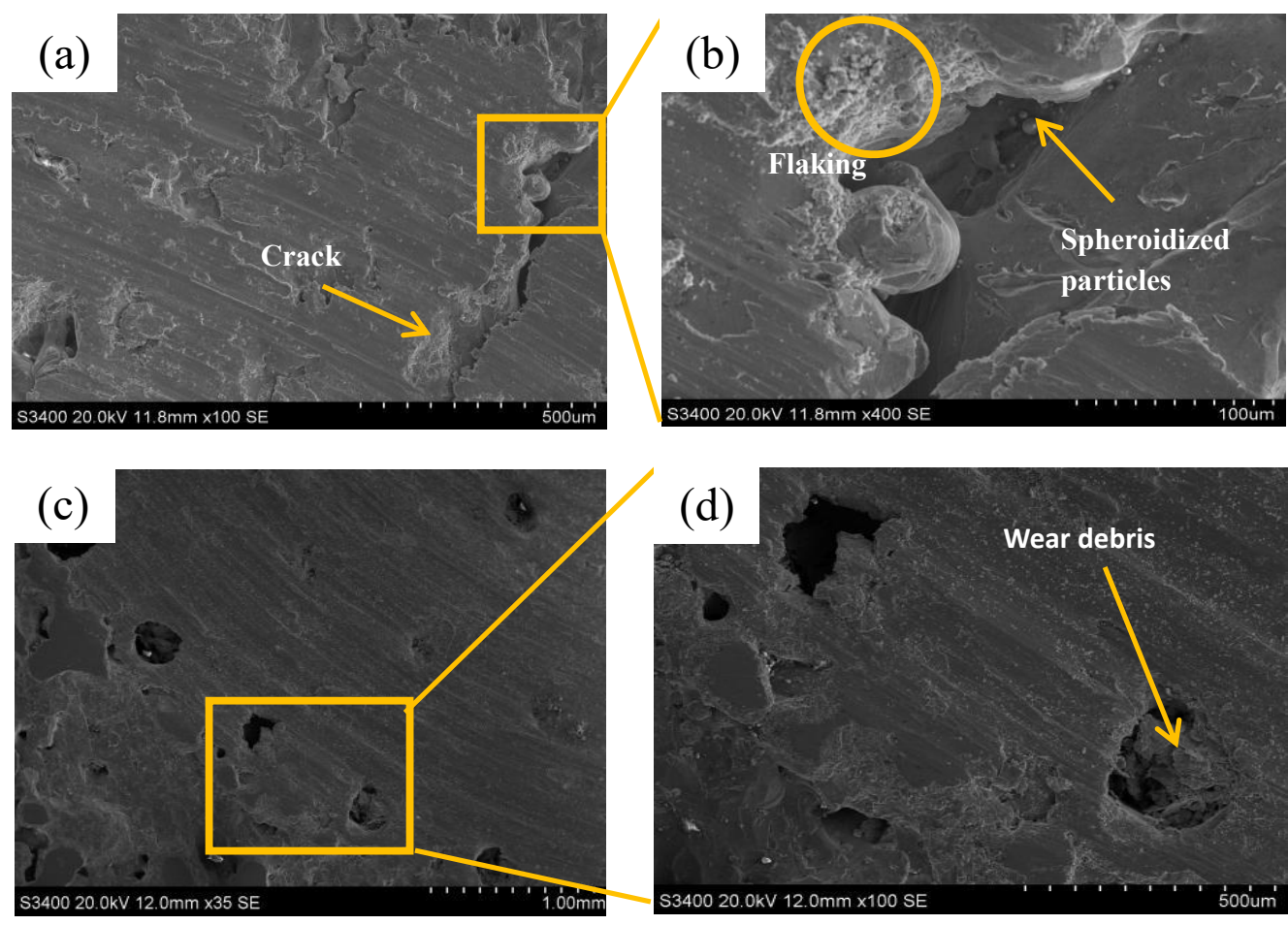

Fig. 8. SEM images of (a) untextured sample; (b) enlarged image of (a); (c) circular textured sample; (d) enlarged image of (c) under dry friction conditions.

In conclusion, the friction coefficient and wear loss of the circular textured sample was small, while the friction coefficient and wear loss of the composite textured sample was the largest. The results showed that the wear resistance of the circular textured sample was the best. Ref. [21] had shown that the surface morphology or the defects had a great effect on the tribological performance of the sample under dry friction conditions, and texture could increase the friction coefficient and reduce the wear loss. The results were basically consistent with the conclusions of circular textured sample obtained in this study. By comparing the friction coefficient of the samples with reduced characteristic size and area ratio of the texture, the relatively large area ratio and characteristic size were helpful in reducing the friction coefficient and wear loss of the 316L SS. Reasonable texture parameters could improve the wear resistance of 316L SS in the dry environment.

\subsection{Tribological performance under simulated body fluid conditions}

The friction coefficients of different textured samples under the PBS solution are shown in Fig. 9. The results indicate that the friction coefficients of the textured samples 
after immersing in the PBS solution for $1 \mathrm{~h}$, are smaller than those under dry condition. This was mainly due to the existence of texture could store more PBS solution, which would amplify the effect of hydrodynamic lubrication under fluid conditions and reduce the friction coefficient. For the samples of group A, the friction coefficient of the composite textured samples was the smallest. It was worth noting that the textured sample had textures for storing wear debris and could provide hydrodynamic lubrication conditions, which led to the friction coefficient of textured sample was significantly less than that of untextured sample. After reducing the textured characteristic size, the friction coefficient change trend of the three textured samples remained the basically same and the friction coefficient rose slightly at the stable stage. The friction coefficient of the composite textured sample reached a minimum of 0.4 , and the friction coefficient of the circular textured samples fluctuated greatly. When the textured area ratio was reduced, the friction coefficients of textured samples were increased significantly. The friction coefficient of the composite textured sample was relatively small and stable, and the friction coefficient of the circular textured sample fluctuated greatly. However, reducing the characteristic size and area ratio of the texture would cause the friction coefficient of the sample to increase, which resulted in a reduction in the effect of the texture to reduce the friction coefficient.

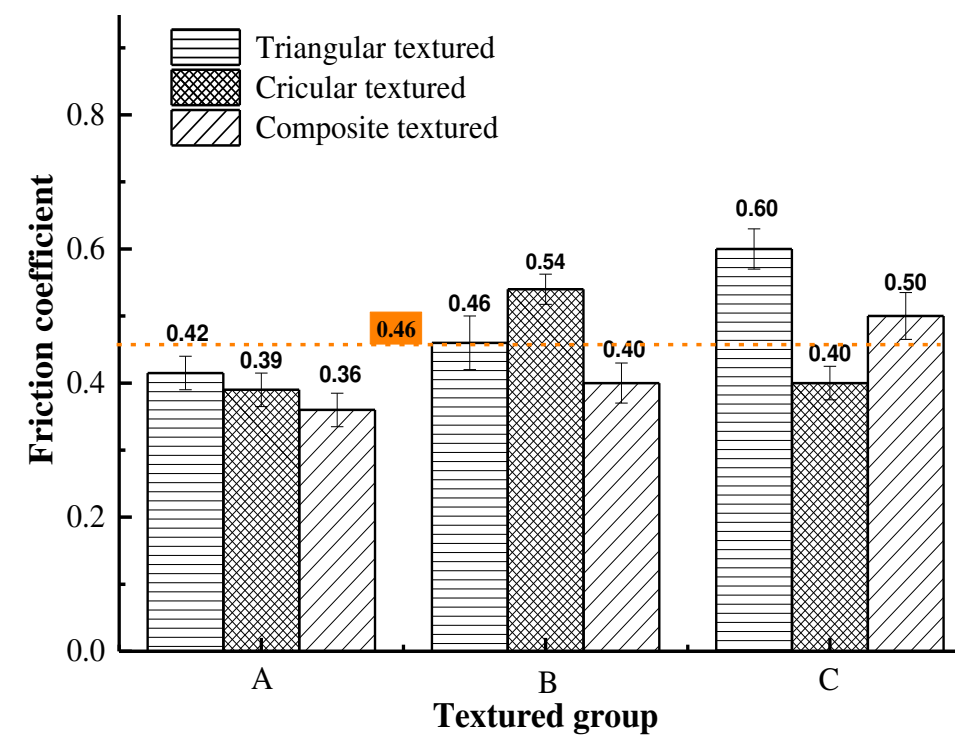

Fig. 9. The friction coefficients of textured samples with different texture parameters under PBS conditions. 
Fig. 10 analyzed the wear loss of samples with different texture parameters under PBS solution. The wear loss of the untextured sample was $11.07 \mathrm{mg}$, which was $15.7 \%$ more than that of the untextured sample under dry friction condition, indicating that the PBS solution would increase the wear loss of the untextured sample. Moreover, it could be found that under PBS condition, the wear loss of the textured samples showed a large reduction overall. The reason is that the PBS solution can provide the prerequisite for the formation of hydrodynamic lubrication. The presence of texture formed a hydrodynamic film to improve the load capacity, and then improved the tribological performance and wear loss of the textured 316L SS sample. In the all groups, the wear loss of the circular textured sample was greater than that of the triangular textured samples. This was because the triangular texture is more effective in forming hydrodynamic lubrication under PBS solution. But for the samples in group B with decreased size, the wear losses of all the three textured samples increase significantly, and the wear loss of the circular textured sample reaches $29.93 \mathrm{mg}$. Simultaneously, when the area ratio of the texture was reduced in group C, the wear loss of the samples increased slightly. It shows that the wear loss and the textured size have a non-linear relationship. Comparing with the area ratio of the texture, the textured characteristic size has a greater impact on the wear loss of the 316L SS under the PBS condition.

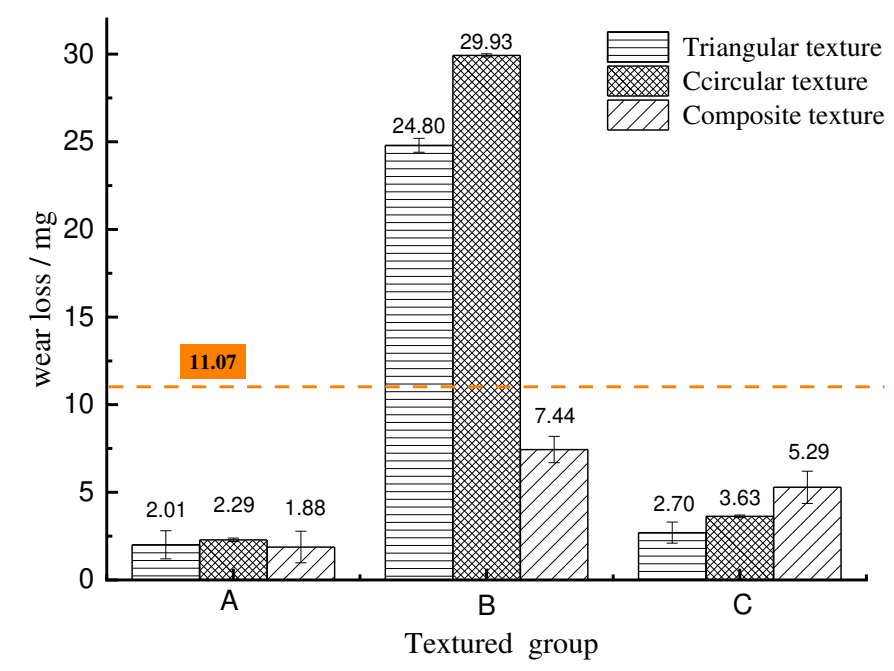

Fig. 10. The wear loss of textured samples with different texture parameters under PBS solution conditions. 
The surface morphology of the samples are observed by SEM, and the results are shown in Fig. 11. It could be seen that there are white patches on the sample surfaces, which was due to the corrosion of 316L SS under the PBS solution (Fig. 11(a)). According to Wang Yue et al. [22], it was found that the white patches on the surface of stainless steel were mainly due to the ferrite segregation and corrosion products caused by more inclusions in the stainless steel material. It could be seen from Fig. 11(b) that the structure of the white corrosion product was relatively loose, and it was easy to fall off the sample surface under the action of friction. Comparing the accumulation of internal wear debris in the texture under dry friction conditions (Fig. 8(c)), the obvious textured morphology could be seen from Fig. 11(c), which indicated that the sample surface is frictional with respect to the surface under dry friction conditions. The damage to the texture was relatively minor under PBS condition. There were obvious flaking and furrows on the sample surface, and small wear debris was stored inside the texture, as shown in Fig. 11(d).
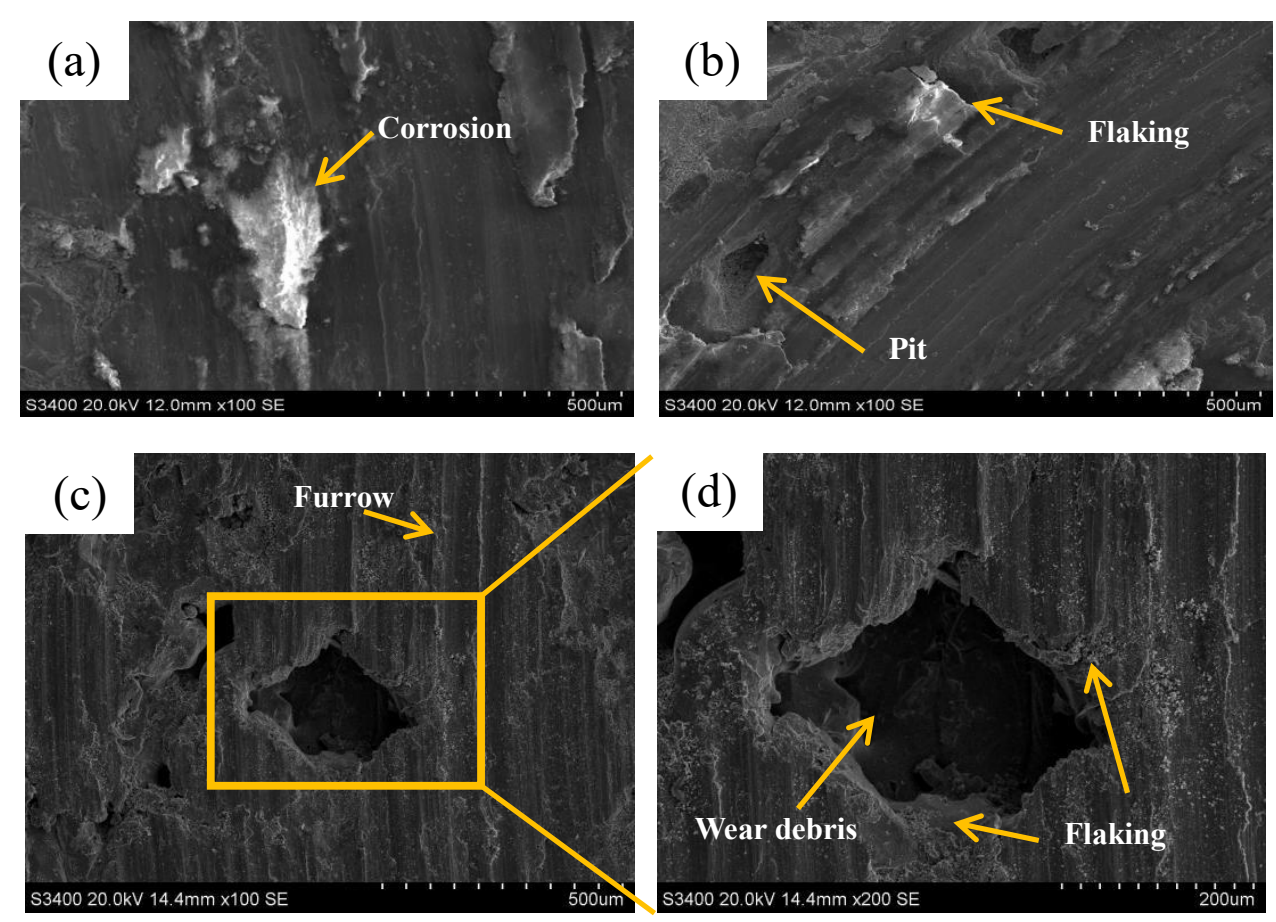

Fig. 11. SEM images of (a) (b) untextured sample in different positions; (c) circular textured sample; (d) enlarged image of (c) under PBS conditions. 


\section{Conclusions}

The tribological performance of textured 316L SS samples prepared by SLM under dry and simulated body fluid lubricated conditions were investigated in this study. The conclusions are as follows:

(1) Under dry conditions, the friction coefficients of the textured samples were higher than that of the untextured sample. The circular textured sample exhibits the lowest friction coefficient, and the composite textured sample exhibits the largest friction coefficient among the textured surfaces. The wear resistance of circular textured samples under dry friction was relatively well. Reducing the textured characteristic size or area ratio will increase the friction coefficient and the wear loss of the $316 \mathrm{~L}$ SS sample.

(2) Under the PBS solution conditions, the friction coefficient of the textured samples in group A are lower than that of untextured sample, and the friction coefficient of the composite textured sample was the lowest. Meanwhile, triangular textured samples have better wear resistance than circular textured samples. While decreased texture size and area ratio will increase the corresponding friction coefficients and wear loss. Especially, reducing the textured characteristic size could lead to a significant increase in the wear loss of the $316 \mathrm{~L}$ SS. Because the surface texture can better exert the hydrodynamic lubrication and store abrasive debris under the PBS lubrication condition, therefore it has the relative smaller wear loss than those under dry condition. By designing reasonable texture parameters can improve the tribological performance of 316L SS medical implants.

\section{Declaration:}

Availability of data and materials: The date that support the finding of this study are available from corresponding author upon reasonable request;

Competing interests: No conflict of interest exits in the submission of this manuscript, and manuscript is approved by all authors for publication;

Funding: National Natural Science Foundation of China (Grant No. 51975042), 
Science and Technology Planning Project of Guangdong Province (2019B010150001) and 2019B010150001 (FRF-TP-19-004A3);

Authors' contributions: Ping Chen: Conceptualization, Methodology; Xiaojie Liu: Validation, Formal analysis, Investigation, Writing - Original Draft; Kangning Jin: Resources, Data Curatio; Xiaoxi Qiao: Writing - Review \& Editing; Weixu Yang: Writing - Review \& Editing.

Acknowledgements: The authors would like to thank National Natural Science Foundation of China (Grant No. 51975042), Science and Technology Planning Project of Guangdong Province (2019B010150001) and 2019B010150001 (FRF-TP-19004A3) for the financial support.

\section{References}

[1] Du Y, Liang H X, Xie D Q, Mao N, Zhao J F, Tian Z J, Wang C J, Shen L D.. 2020 Materials Chemistry and Physics 239: 121968

[2] Ataee A, Li Y C, Wen C E. 2019, Acta Biomaterialia 97: 587-596

[3] Tridello A, Fiocchi J, Biffi C A, Chiandussi G, Rossetto M, Tuissi A, Paolino D S. 2019, Procedia Structural Integrity 18: 314-321

[4] Man C, Dong C F, Liu T T, Kong D C, Wang D K, Li X G. 2019 Applied Surface Science 467-468: 193-20

[5] Bartolomeu F, Buciumeanu M, Pinto E, Alves N, Carvalho O, Silva F S, Miranda G. 2017, Additive Manufacturing 16: 81-89

[6] Andersen K N, Bienk E J, Schweitz K O, Reitz H, Chevallier J, Kringhøj P, Bøttiger J. Deposition, 2000, Surface and Coatings Technology 123: 219-226

[7] Katta P P, Nalliyan R. 2019, Surface and Coatings Technology 375: 715-726

[8] Kong D, Ni X, Dong C, Lei X, Zhang L, Man C, Yao J, Cheng X, Li X. 2018, Materials and Design 152: 88-101

[9] Zhu Y, Zou J, Chen X, Yang H. 2016. Wear 350-351: 46-55

[10] Sun Y, Moroz A, Alrbaey K. 2014, Journal of Materials Engineering and Performance 23(2): 518-526

[11] Zhu Y, Lin G L, Khonsari M M, Zhang J H, Yang H. 2018, Journal of Materials Processing Technology 262:41-52

[12] Amanov A, Lee S W, Pyun Y S. 2017, Materials Science and Engineering: C 71: $176-185$

[13] Chen P, Liu X J, Huang M J, Shan B. 2019, Tribology International 132: 244-252 [14] Fu Y H, Yang S J, Xu H, Hua X J, Wang Z L.2014, Journal of Jiangsu University (Natural Science Edition) 35(3): 343-348

[15] Zhang H, Hua M, Dong G N, Zhang D Y, Chin K S. 2016, Tribology International 93:583-592

[16] Segu D Z, Kim J H, Choi S G, Jung Y S, Kim S S. 2013, Surface and Coatings 
Technology 232:504-514

[17] Segu D Z, Choi S G, Choi J H, Kim S S.2013, Applied Surface Science 270:58-63 [18]Powders M, Flowmeter H, Spectrometry F, Steels A, Methods S, Media S, Metals R R, Coupled I, Dispersive W, Spectrometry X F, 2014, West Conshohocken, ASTM [19] Wang P, Shi W T, Liu Y D, Qi B, Yang J, Zhang F F, Ma Y Y. 2019, Special Casting and Nonferrous Alloys 39(05):501-505

[20] Lu C Y, Yang X F, Wang S R, Wang Y J, Wu Y B, Chen H L2018, Tribology 38(05):537-546

[21] Li J B, Liu S, Yu A B, Xiang S T. 2018, Tribology International 118:37-45

[22] Wang Y, Li J L.2017, Heat Treatment of Metals 42(01):183-186 